\title{
Risk Factors for Acute Hemorrhagic Rectal Ulcer Syndrome and Its Prognosis: A Density Case-Control Study
}

\author{
Toshihiko Komai, ${ }^{1,2}$ Fumio Omata $\mathbb{D D}^{1}{ }^{1}$ Yasutoshi Shiratori, ${ }^{1}$ Daiki Kobayashi $\mathbb{D}^{1}{ }^{1}$ \\ and Hiroko Arioka ${ }^{1}$ \\ ${ }^{1}$ Department of Internal Medicine, St. Luke's International University, 9-1 Akashi-cho, Chuo-ku, Tokyo 104-8560, Japan \\ ${ }^{2}$ Department of Allergy and Rheumatology, Graduate School of Medicine, The University of Tokyo, 7-3-1 Hongo, Bunkyo-ku, \\ Tokyo 113-8655, Japan
}

Correspondence should be addressed to Fumio Omata; omataf@icloud.com

Received 31 January 2018; Revised 7 May 2018; Accepted 7 June 2018; Published 8 August 2018

Academic Editor: Paul Enck

Copyright (@ 2018 Toshihiko Komai et al. This is an open access article distributed under the Creative Commons Attribution License, which permits unrestricted use, distribution, and reproduction in any medium, provided the original work is properly cited.

\begin{abstract}
Acute hemorrhagic rectal ulcer syndrome (AHRUS) can cause fatal gastrointestinal bleeding. However, there have been few epidemiological studies investigating risk factors of AHRUS. To determine the risk factors and predict one-year survival after onset of AHRUS, we conducted a retrospective density case-control study in a tertiary referral hospital. Patients with hematochezia, bloody stool, and rectal ulcer confirmed by colonoscopy between 2003 and 2011 were diagnosed as AHRUS ( $n=$ 38). Patients with malignancies, infectious colitis, ulcerative colitis, or solitary rectal ulcer syndrome were excluded. Control subjects $(n=123)$ without rectal ulcer were selected by risk set sampling for each AHRUS. Multivariate logistic regression analyses revealed that the significant adjusted odds ratio (95\% confidence interval) of hospitalization, antithrombotic drug use, and one gram increase of serum albumin was 15.7 (2.25-108.9), 12.1 (1.53-94.4), and 0.11 (0.02-0.52), respectively. Endoscopic hemostasis for rectal bleeding was performed in 8 cases $(21 \%)$. Seventeen percent of patients died within one year after the episode of AHRUS from non-AHRUS causes. This study revealed that hospitalization, antithrombotic drug use, and lower serum albumin value were significant risk factors for AHRUS, and that AHRUS was an unfavorable prognostic condition. This information could be helpful in recognizing high-risk patients of rectal bleeding and applying preventive measures.
\end{abstract}

\section{Introduction}

Rectal ulcer, unrelated to malignancy, inflammatory bowel diseases (IBD), or infectious colitis includes two distinct disease entities: solitary rectal ulcer syndrome (SRUS) $[1,2]$ and acute hemorrhagic rectal ulcer syndrome (AHRUS) [3]. SRUS is a chronic benign disorder, most common in young adults, often associated with bowel disturbances, abnormal defecation, and mucosal prolapse $[4,5]$. AHRUS is characterized by sudden massive rectal bleeding, most often in elderly patients with underlying comorbidities [6,7]. AHRUS has been reported to be the most common cause of acute lower gastrointestinal bleeding in hospitalized patients with comorbidities $[3,6,7]$.

Fecal evacuation disorder was reported to be a potential risk factor for SRUS [8-10], and gut-directed biofeedback therapy is an effective behavioral intervention $[8,9]$. However, there have been few studies investigating risk factors and prophylactic interventions of life-threatening AHRUS. This lack of information may contribute to delay in making the diagnosis and in instituting preventive measures. In addition, there has been no report of survival analysis of the patients suffering from AHRUS. The aims of our study were to determine risk factors of AHRUS and to predict its oneyear survival.

\section{Materials and Methods}

2.1. Study Population. A total of 23,988 colonoscopies were performed in a tertiary referral hospital in Tokyo, Japan, from 2003 to 2011. Thirty-eight cases with the diagnosis of AHRUS [3, 6] were identified after excluding associated 


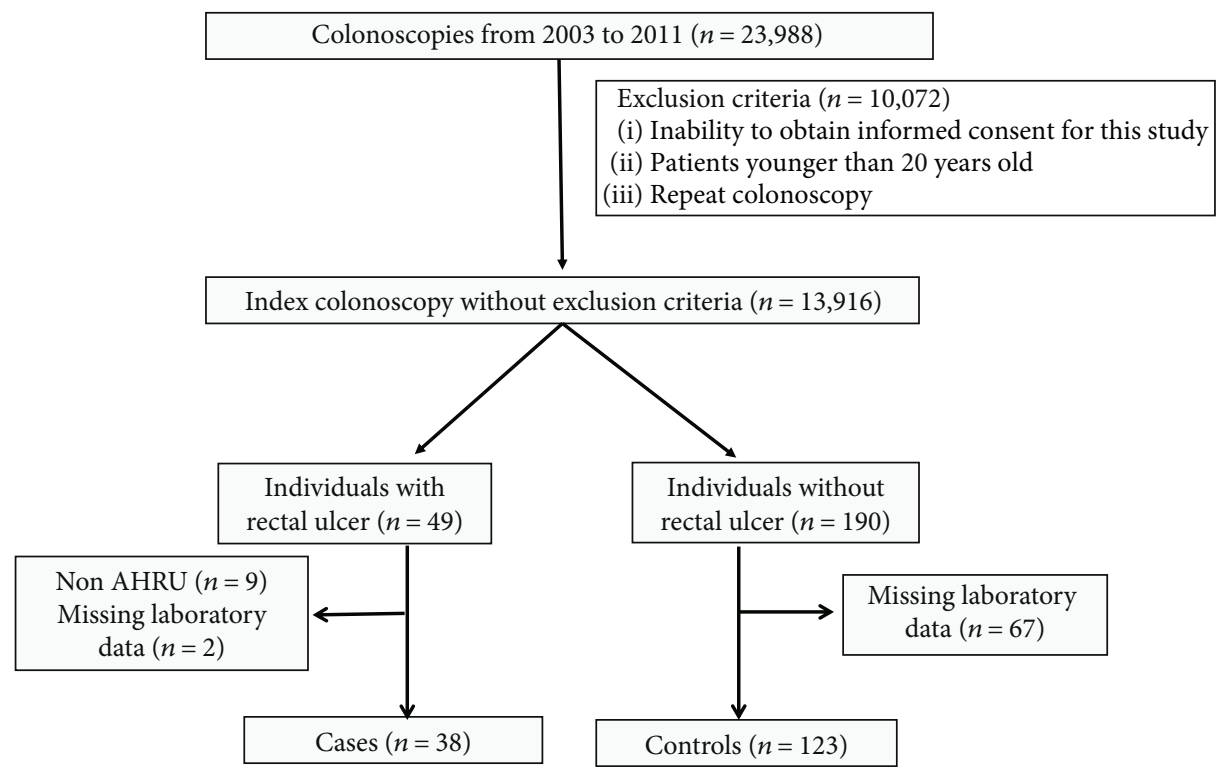

Figure 1: Study flow diagram depicting selection of cases and controls. 13,916 index colonoscopies out of 23,988 were performed in the patients older than 20 years of age in a tertiary medical center in Tokyo, Japan. Thirty-eight acute hemorrhagic rectal ulcer syndrome patients were diagnosed. From the same database of index colonoscopies, 123 patients without rectal ulcer were selected as a control group by risk set sampling.

ulcerative colitis $(n=3)$, infectious colitis $(n=1)$, rectal ulcer with no gastrointestinal bleeding $(n=4)$, SRUS $(n=1)$, and lack of laboratory data $(n=2)$. Subjects who had not given informed consent for use of their electronic records and subjects under 20 years of age were also excluded. We waived informed consent from patients who were included in our study.

One hundred twenty-three subjects without rectal ulcer and with adequate laboratory values were selected as controls by risk set sampling $[11,12]$ (Figure 1). When the laboratory values of controls were missing on the same day of colonoscopy, their laboratory values within 6 months after colonoscopy were imputed. This study was approved by St. Luke's International University Research Ethics Committee (authorization number: 11-R162).

2.2. Data Availability. The data of this study were handled with all of the authors under strict control, and availability was restricted for ethical reasons. However, anonymized data could be available for other researchers upon request with the permission of our ethical committee.

2.3. Diagnosis of Rectal Ulcer. The diagnosis of rectal ulcer in cases and confirmation of no rectal ulcer in controls were established with colonoscopy. AHRUS was defined as ulcer associated with hematochezia or bloody stool, whereas rectal ulcer was present at colonoscopy after excluding other diseases which might cause rectal ulcer $[3,6]$. The location of ulcers in the rectum was classified like that used for colorectal cancer: rectosigmoid (Rs), rectum above the peritoneal reflection $(\mathrm{Ra})$, or rectum below the peritoneal reflection $(\mathrm{Rb})$.

2.4. Candidate Risk Factors for AHRUS. We investigated whether age, gender, comorbidities, laboratory values, hospitalization, and antithrombotic use were associated with AHRUS.

2.5. Statistical Analyses. Fisher's exact test was applied for proportion. Student's $t$-test or Wilcoxon rank sum test was used for continuous variables. Bivariate and multivariate logistic regressions were used to calculate odds ratio (OR). Variables with $P$ value less than 0.2 in bivariate logistic regression were used in multivariate analyses. $P$ value less than 0.05 was considered statistically significant. KaplanMeier estimates were used for survival analysis. All 95\% confidence intervals were two-sided. All analyses were conducted with $\mathrm{JMP}^{\circledR}$ version 13 statistical software (SAS Institute, Cary, NC). In conducting this study, we followed the checklist of items for case-control study described in strengthening the reporting of observational studies in epidemiology (STROBE) statement [13].

\section{Results}

3.1. Characteristics of Patients with AHRUS. Table 1 presents patients' characteristics, candidate risk factors for AHRUS, laboratory data, and the results of bivariate analyses. Age, hospitalization, antithrombotic drug use, comorbidities (hypertension, ischemic heart disease, and cerebrovascular disease), and laboratory findings (serum albumin, serum creatinine, white blood cell count, and hemoglobin levels) were significantly different between cases and controls. Enemas were not used in the enrolled participants.

More than half of the rectal ulcer was located in $\mathrm{Rb}$ (Figure 2). We performed biopsy in eleven patients. The histological findings of all these patients were nonspecific and did not show any finding suggesting SRUS or IBD. 
TABLe 1: Patients' characteristics and bivariate analyses.

\begin{tabular}{|c|c|c|c|}
\hline Variable & Cases & Controls & $P$ value \\
\hline Age (years), mean (SD) & $76(12)$ & $60(15)$ & $<0.0001$ \\
\hline Sex, male, $n(\%)$ & $18(47.4)$ & $62(50.4)$ & 0.85 \\
\hline Hospitalization, $n(\%)$ & $32(84.2)$ & $14(11.4)$ & $<0.0001$ \\
\hline Hospitalization period (day), mean (SD) & $19.0(24.4)$ & $1.0(4.9)$ & $<0.0001$ \\
\hline Usage of antithrombotic drugs, $n(\%)$ & $25(65.8)$ & $16(13.0)$ & $<0.0001$ \\
\hline \multicolumn{4}{|l|}{ Comorbidity } \\
\hline Hypertension, $n(\%)$ & $23(60.5)$ & $45(36.6)$ & 0.014 \\
\hline Diabetes mellitus, $n(\%)$ & $10(26.3)$ & $19(15.5)$ & 0.15 \\
\hline Ischemic heart disease, $n(\%)$ & $13(34.2)$ & $5(4.1)$ & $<0.0001$ \\
\hline Cerebral vascular disease, $n(\%)$ & $17(44.7)$ & $8(6.5)$ & $<0.0001$ \\
\hline Under hemodialysis, $n(\%)$ & $4(10.5)$ & $3(2.44)$ & 0.054 \\
\hline Malignancies, $n(\%)$ & $6(15.8)$ & $30(24.4)$ & 0.37 \\
\hline \multicolumn{4}{|l|}{ Laboratory findings } \\
\hline Serum albumin (mg/dl), mean (SD) & $2.67(0.60)$ & $4.09(0.52)$ & $<0.0001$ \\
\hline Serum Cr (mg/dl), mean (SD) & $1.35(1.50)$ & $1.16(2.21)$ & 0.63 \\
\hline Serum AST (U/l), mean (SD) & $26.0(14.6)$ & $22.8(9.9)$ & 0.13 \\
\hline Serum ALT (U/l), mean (SD) & $25.3(19.9)$ & $23.3(21.8)$ & 0.62 \\
\hline White blood cell count (cells/ $\mu \mathrm{l})$, mean (SD) & $8013(4757)$ & $5959(2047)$ & 0.0002 \\
\hline Hemoglobin (g/dl), mean (SD) & $9.51(2.09)$ & $12.7(2.06)$ & $<0.0001$ \\
\hline Platelet count (thousand cells/ $\mu \mathrm{l}$ ), mean (SD) & $247.3(132.4)$ & $236.7(90.8)$ & 0.58 \\
\hline
\end{tabular}

SD: standard deviation; AST: aspartate aminotransferase; ALT: alanine aminotransferase.

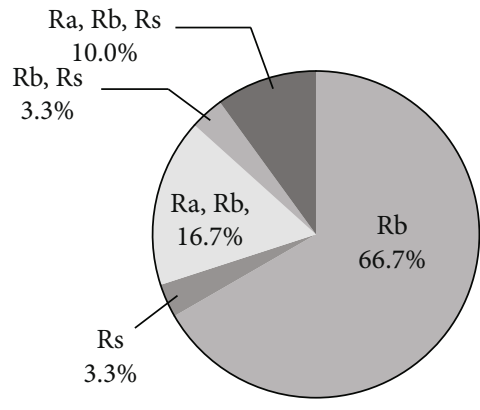

FIGURE 2: Pie chart depicting the location of rectal ulcer in 30 of 38 patients with acute hemorrhagic rectal ulcer syndrome (in 8 cases, rectal ulcer location was not described in endoscopy report). Rs: rectosigmoid; $\mathrm{Ra}$ : rectum above the peritoneal reflection; $\mathrm{Rb}$ : rectum below the peritoneal reflection.

3.2. Density Case-Control Analysis for the Identification of Risk Factors for AHRUS. In bivariate logistic regression analyses, age, hospitalization, antithrombotic drug use; the comorbidities of ischemic heart disease, cerebrovascular disease, and chronic kidney disease under hemodialysis; and the laboratory findings of white blood cell count, hemoglobin, and serum albumin values were significantly associated with AHRUS.

In multivariate logistic regression analyses, hospitalization (adjusted OR 15.65, 95\% CI 2.25-108.9), antithrombotic drug use (adjusted OR 12.05, 95\% CI 1.53-94.4), and one gram decrease of serum albumin (adjusted OR 0.11, 95\% CI 0.02-0.52) were significantly associated with AHRUS. On the other hand, neither of age, the indicated comorbidities were not significant risk factors (Table 2).

3.3. Outcome and Long-Term Survival of Rectal Bleeding. Endoscopic treatment for attempted control of bleeding (clipping or band ligation) was performed in 8 of the 38 patients $(21 \%)$, as listed in Table 3 . Rebleeding occurred in two patients and was treated successfully with reclipping. Sixteen of the 38 patients ( $42 \%$; 95\% CI $28-58 \%$ ) needed blood transfusion. All patients received hydration and restriction of oral intake. We did not use sucralfate enema in any patient.

Survival analysis showed that $17 \%$ of patients died within one year after their rectal bleeding episode from causes not related to their AHRUS (Figure 3). These results collectively indicated that AHRUS could be an unfavorable prognostic condition, and the preventive intervention based on the risk factors would be necessary.

Among 33 survivors at one year, follow-up colonoscopy at least 30 days after rectal bleeding was performed in four patients; complete rectal ulcer healing was confirmed in all these patients.

\section{Discussion}

This study is the first case-control study that we are aware of about risk factors for AHRUS. We found that hospitalization, antithrombotic drug use, and hypoalbuminemia were significant risk factors for developing AHRUS. Seventeen percent of AHRUS patients died within one year of causes not related to the AHRUS. 
TABLE 2: Bivariate and multivariate logistic regression analyses.

\begin{tabular}{|c|c|c|c|c|c|c|}
\hline Variable & Crude OR & $95 \% \mathrm{CI}$ & $P$ value & Adjusted OR & $95 \% \mathrm{CI}$ & $P$ value \\
\hline Age & 1.10 & $1.06-1.14$ & $<0.0001$ & 1.03 & $0.96-1.11$ & 0.36 \\
\hline Hospitalization & 41.52 & $14.8-116.8$ & $<0.0001$ & 15.65 & $2.25-108.9$ & 0.006 \\
\hline Antithrombotic drug use & 12.86 & $5.49-30.13$ & $<0.0001$ & 12.05 & $1.53-94.4$ & 0.018 \\
\hline \multicolumn{7}{|l|}{ Comorbidity } \\
\hline Hypertension & 2.66 & $1.26-5.61$ & 0.0093 & 0.51 & $0.08-3.18$ & 0.47 \\
\hline Diabetes mellitus & 0.13 & $0.82-4.68$ & 0.141 & 1.33 & $0.12-14.8$ & 0.82 \\
\hline Ischemic heart disease & 12.27 & $4.01-37.53$ & $<0.0001$ & 8.44 & $0.89-80.3$ & 0.063 \\
\hline Cerebral vascular disease & 11.64 & $4.45-30.40$ & $<0.0001$ & 2.41 & $0.42-13.7$ & 0.32 \\
\hline On hemodialysis & 4.71 & $1.00-22.05$ & 0.049 & 0.66 & $0.01-33.5$ & 0.84 \\
\hline \multicolumn{7}{|l|}{ Laboratory findings } \\
\hline White blood cell count & 1.26 & $1.09-1.46$ & 0.0004 & 1.24 & $0.94-1.63$ & 0.11 \\
\hline Hemoglobin & 0.53 & $0.43-0.66$ & $<0.0001$ & 0.97 & $0.59-1.60$ & 0.9 \\
\hline Serum AST & 1.02 & $0.99-1.05$ & 0.145 & 0.98 & $0.92-1.05$ & 0.64 \\
\hline Serum albumin & 0.036 & $0.01-0.10$ & $<0.0001$ & 0.11 & $0.02-0.52$ & 0.006 \\
\hline
\end{tabular}

OR: odds ratio; AST: aspartate aminotransferase.

TABle 3: Endoscopic hemostatic procedure.

\begin{tabular}{lc}
\hline Procedures and rebleeding rate & Cases \\
\hline Hemostatic procedure, $n(\%)$ & $8(21)$ \\
Band ligation, $n$ & 2 \\
Clipping, $n$ & 6 \\
Rebleeding after hemostatic procedure, $n(\%)$ & $2(25)$ \\
\hline
\end{tabular}

Rectal ulcers with massive hemorrhage in critically ill patients have long been recognized $[6,14,15]$, but the infrequency and fatal clinical course has precluded clinical studies about its risk factors and establishing this condition as an independent clinical disease. Recently, however, rectal ulcers with massive hemorrhage have been recognized as an emerging clinical entity, AHRUS [3, 6, 7, 16].

Previous observational studies have reported the characteristics of AHRUS patients, such as older age, immobility, antithrombotic drug use, and comorbidities such as diabetes mellitus, coronary artery diseases, cerebrovascular attacks, sepsis, liver failure [3], hypoalbuminemia [17-19], and chronic renal failure with hemodialysis [15]. The characteristics of cases in our research were compatible with those in observational studies and supported etiological assumptions except for older age [17-20]. Like others $[6,7]$, we found that AHRUS patients often require hemostatic procedures and blood transfusion. We did not use sucralfate enemas in our patients, although a case series [21] reported it effective.

Several studies have shown that serum hypoalbuminemia can be an independent risk factor for decreased microperfusion and pressure ulcers because albumin helps maintain oncotic pressure and vascular refilling [22-24]. Our results were compatible with the results of the previous observational study, which suggested that hypoalbuminemia and high blood urea nitrogen levels were risk factors for lower gastrointestinal bleeding, mainly due to ischemic colitis and rectal ulcer in critically ill patients [19].

It seems reasonable that immobility during hospitalization, hypoperfusion of local rectal blood flow in the elderly, and hypoalbuminemia could lead to the formation of rectal ulcer; previous epidemiological studies suggested that these could be risk factors of SRUS or stercoral ulcer $[2,9,10]$. Our endoscopic findings indicated that more than $90 \%$ of rectal ulcers were located in $\mathrm{Rb}$, which implied specific local vascular flow disturbance.

Baseline blood flow in the rectal mucosa, measured by laser Doppler flowmetry, has been found significantly below normal in patients with SRUS [9] or AHRUS [25], and the blood flow is significantly reduced in the horizontal supine position at bed rest. Baroreceptor-mediated vasoconstriction in hypovolemic conditions [26] or in bedridden, elderly, or hospitalized patients has been reported to cause ischemic proctitis [27].

Although ischemia might be involved in both SRUS and AHRUS, the pathogenesis of the two clinical entities seems different. Comparing with patients with SRUS associated with fecal evacuation disorders [10], the higher prevalence of AHRUS among immobilized older patients with cardiovascular risks $[6,7]$ suggests that nutrient blood flow is disturbed in AHRUS, rather than there is local or direct pressure in the rectum as in SRUS.

Our study has limitations and strengths. First, we did not assess the possible role of constipation or overactivity of the anal sphincter in causing rectal ulceration or ischemia because of retrospective design. Second, there could be selection bias by excluding subjects with missing laboratory values; this bias might have an effect toward the null on the odds ratio of hospitalization for AHRS. Despite these factors, our study was valuable because this was the first density case-control study in Japan to explore the comprehensive risk factors for the occurrence of AHRUS and patients' survival after AHRUS. 


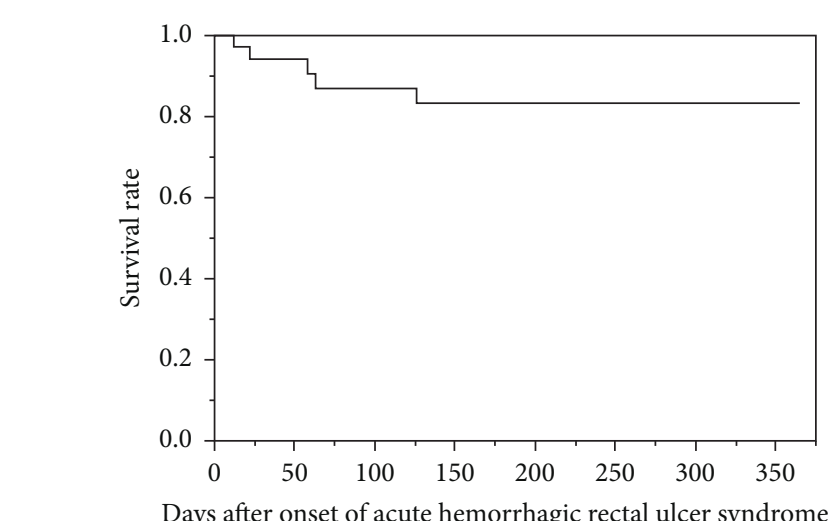

Number at risk 38 22

Figure 3: Kaplan-Meier estimates after onset of acute rectal hemorrhagic rectal ulcer. 17 percent of patients with acute hemorrhagic rectal ulcer syndrome (AHRUS) died of non-AHRUS problem in one year.

\section{Conclusions}

Hospitalization, antithrombotic drug use, and hypoalbuminemia were significant risk factors for AHRUS. Knowledge of these risk factors could make clinicians more alert to the possibility of AHRUS being the cause of lower gastrointestinal bleeding, to take measures to prevent it and to perform proctoscopy or colonoscopy promptly to diagnose it. Onset of AHRUS has an unfavorable prognosis.

\section{Data Availability}

The data used to support the findings of this study are available from the corresponding author upon request.

\section{Conflicts of Interest}

The authors declare that there is no conflict of interest regarding the publication of this paper.

\section{Acknowledgments}

The authors thank Professor Emeritus William R. Brown, University of Colorado School of Medicine, USA, for a critical review of the manuscript.

\section{References}

[1] N. R. Womack, N. S. Williams, J. H. Holmfield, and J. F. Morrison, "Pressure and prolapse-the cause of solitary rectal ulceration," Gut, vol. 28, no. 10, pp. 1228-1233, 1987.

[2] Q. C. Zhu, R. R. Shen, H. L. Qin, and Y. Wang, "Solitary rectal ulcer syndrome: clinical features, pathophysiology, diagnosis and treatment strategies," World Journal of Gastroenterology, vol. 20, no. 3, pp. 738-744, 2014.

[3] C. A. Tseng, L. T. Chen, K. B. Tsai et al., “Acute hemorrhagic rectal ulcer syndrome: a new clinical entity? Report of 19 cases and review of the literature," Diseases of the Colon and Rectum, vol. 47, no. 6, pp. 895-905, 2004.
[4] A. I. Sharara, C. Azar, S. S. Amr, M. Haddad, and M. A. Eloubeidi, "Solitary rectal ulcer syndrome: endoscopic spectrum and review of the literature," Gastrointestinal Endoscopy, vol. 62, no. 5, pp. 755-762, 2005.

[5] J. J. Tjandra, V. W. Fazio, J. M. Church, I. C. Lavery, J. R. Oakley, and J. W. Milsom, "Clinical conundrum of solitary rectal ulcer," Diseases of the Colon and Rectum, vol. 35, no. 3, pp. 227-234, 1992.

[6] C. K. Lin, C. C. Liang, H. T. Chang, F. M. Hung, and T. H. Lee, "Acute hemorrhagic rectal ulcer: an important cause of lower gastrointestinal bleeding in the critically ill patients," Digestive Diseases and Sciences, vol. 56, no. 12, pp. 3631-3637, 2011.

[7] K. Takeuchi, Y. Tsuzuki, T. Ando et al., "Clinical characteristics of acute hemorrhagic rectal ulcer," Journal of Clinical Gastroenterology, vol. 33, no. 3, pp. 226-228, 2001.

[8] S. S. C. Rao, R. Ozturk, S. De Ocampo, and M. Stessman, "Pathophysiology and role of biofeedback therapy in solitary rectal ulcer syndrome," The American Journal of Gastroenterology, vol. 101, no. 3, pp. 613-618, 2006.

[9] M. E. Jarrett, A. V. Emmanuel, C. J. Vaizey, and M. A. Kamm, "Behavioural therapy (biofeedback) for solitary rectal ulcer syndrome improves symptoms and mucosal blood flow," Gut, vol. 53, no. 3, pp. 368-370, 2004.

[10] A. Sharma, A. Misra, and U. C. Ghoshal, "Fecal evacuation disorder among patients with solitary rectal ulcer syndrome: a case-control study," Journal of Neurogastroenterology and Motility, vol. 20, no. 4, pp. 531-538, 2014.

[11] B. Langholz and L. Goldstein, "Risk set sampling in epidemiologic cohort studies," Statistical Science, vol. 11, no. 1, pp. 3553, 1996.

[12] K. J. Rothman, Epidemiology. An Introduction, Boson: Oxford University Press, 2002.

[13] E. von Elm, D. G. Altman, M. Egger, S. J. Pocock, P. C. Gøtzsche, and J. P. Vandenbroucke, "The strengthening the reporting of observational studies in epidemiology (STROBE) statement: guidelines for reporting observational studies," The Lancet, vol. 370, no. 9596, pp. 1453-1457, 2007.

[14] M. Goldberg, G. C. Hoffman, and D. G. Wombolt, "Massive hemorrhage from rectal ulcers in chronic renal failure," Annals of Internal Medicine, vol. 100, no. 3, p. 397, 1984.

[15] F. Kanwal, G. Dulai, D. M. Jensen et al., "Major stigmata of recent hemorrhage on rectal ulcers in patients with severe hematochezia: endoscopic diagnosis, treatment, and outcomes," Gastrointestinal Endoscopy, vol. 57, no. 4, pp. 462468, 2003.

[16] S. B. Almadani and M. Brown, "Acute hemorrhagic rectal ulcer syndrome (AHRUS): an under-recognized condition," The American Journal of Gastroenterology, vol. 108, p. S362, 2013.

[17] T. Hotta, K. Takifuji, S. Tonoda et al., "Risk factors and management for massive bleeding of an acute hemorrhagic rectal ulcer," The American Surgeon, vol. 75, no. 1, pp. 6673, 2009.

[18] B. C. Kim, J. H. Cheon, T. I. Kim, and W. H. Kim, "Risk factors and the role of bedside colonoscopy for lower gastrointestinal hemorrhage in critically ill patients," Hepato-Gastroenterology, vol. 55, no. 88, pp. 2108-2111, 2008.

[19] J. H. Jung, J. W. Kim, H. W. Lee et al., “Acute hemorrhagic rectal ulcer syndrome: comparison with non-hemorrhagic rectal ulcer lower gastrointestinal bleeding," Journal of Digestive Diseases, vol. 18, no. 9, pp. 521-528, 2017. 
[20] T. Oku, M. Maeda, H. Ihara et al., "Clinical and endoscopic features of acute hemorrhagic rectal ulcer," Journal of Gastroenterology, vol. 41, no. 10, pp. 962-970, 2006.

[21] S. A. Zargar, M. S. Khuroo, and R. Mahajan, "Sucralfate retention enemas in solitary rectal ulcer," Diseases of the Colon and Rectum, vol. 34, no. 6, pp. 455-457, 1991.

[22] S. Iizaka, H. Sanada, Y. Matsui et al., "Serum albumin level is a limited nutritional marker for predicting wound healing in patients with pressure ulcer: two multicenter prospective cohort studies," Clinical Nutrition, vol. 30, no. 6, pp. $738-$ $745,2011$.

[23] E. Mistrík, S. Dusilová-Sulková, V. Bláha, and L. Sobotka, "Plasma albumin levels correlate with decreased microcirculation and the development of skin defects in hemodialyzed patients," Nutrition, vol. 26, no. 9, pp. 880-885, 2010.

[24] R. Serra, S. Caroleo, G. Buffone et al., "Low serum albumin level as an independent risk factor for the onset of pressure ulcers in intensive care unit patients," International Wound Journal, vol. 11, no. 5, pp. 550-553, 2014.

[25] S. Nakamura, K. Okawa, J. Hara et al., "Etiology of acute hemorrhagic rectal ulcer laser Doppler analysis of rectal mucosal blood flow in lateral and horizontal supine position at bed rest," Gastroenterological Endoscopy, vol. 38, pp. 1481-1487, 1996.

[26] A. Thorén, S. E. Ricksten, S. Lundin, B. Gazelius, and M. Elam, "Baroreceptor-mediated reduction of jejunal mucosal perfusion, evaluated with endoluminal laser Doppler flowmetry in conscious humans," Journal of the Autonomic Nervous System, vol. 68, no. 3, pp. 157-163, 1998.

[27] A. E. Bharucha, W. J. Tremaine, C. D. Johnson, and K. P. Batts, "Ischemic proctosigmoiditis," The American Journal of Gastroenterology, vol. 91, no. 11, pp. 2305-2309, 1996. 


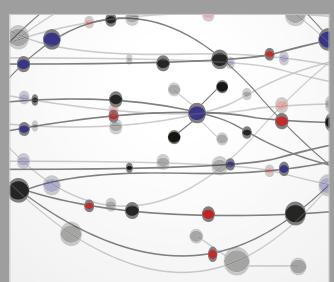

The Scientific World Journal
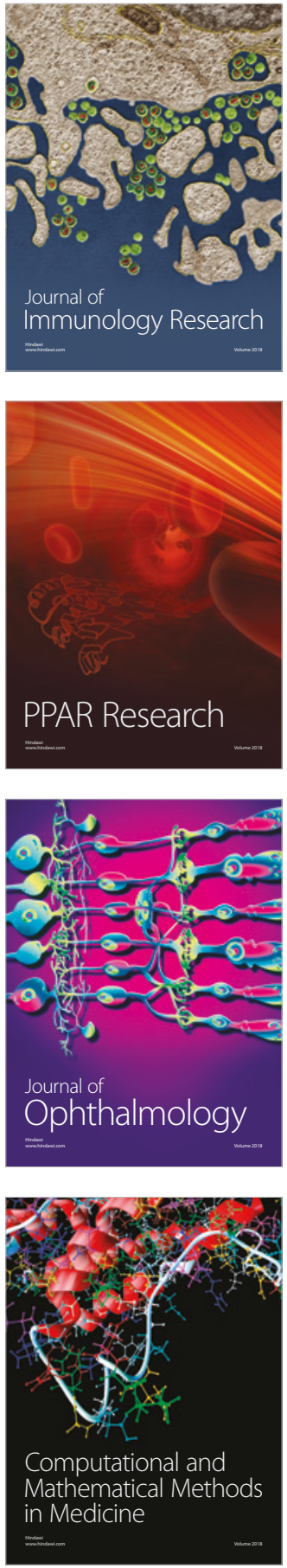

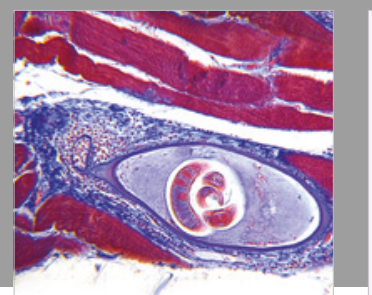

Gastroenterology Research and Practice

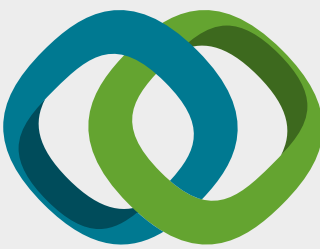

\section{Hindawi}

Submit your manuscripts at

www.hindawi.com
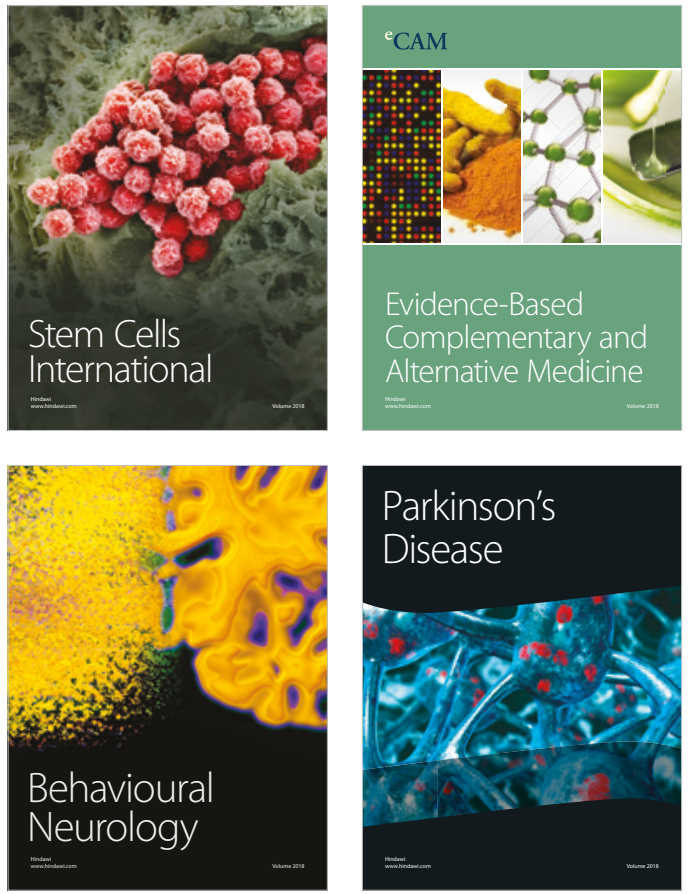

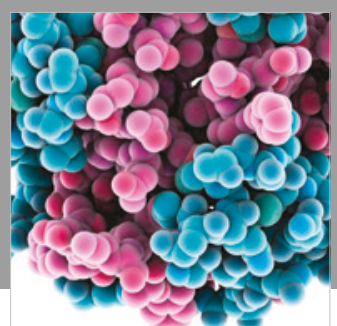

ournal of

Diabetes Research

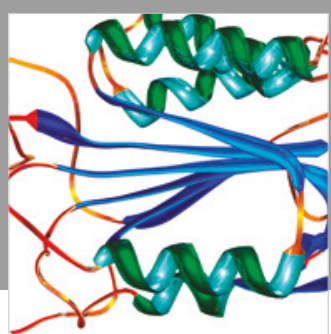

Disease Markers
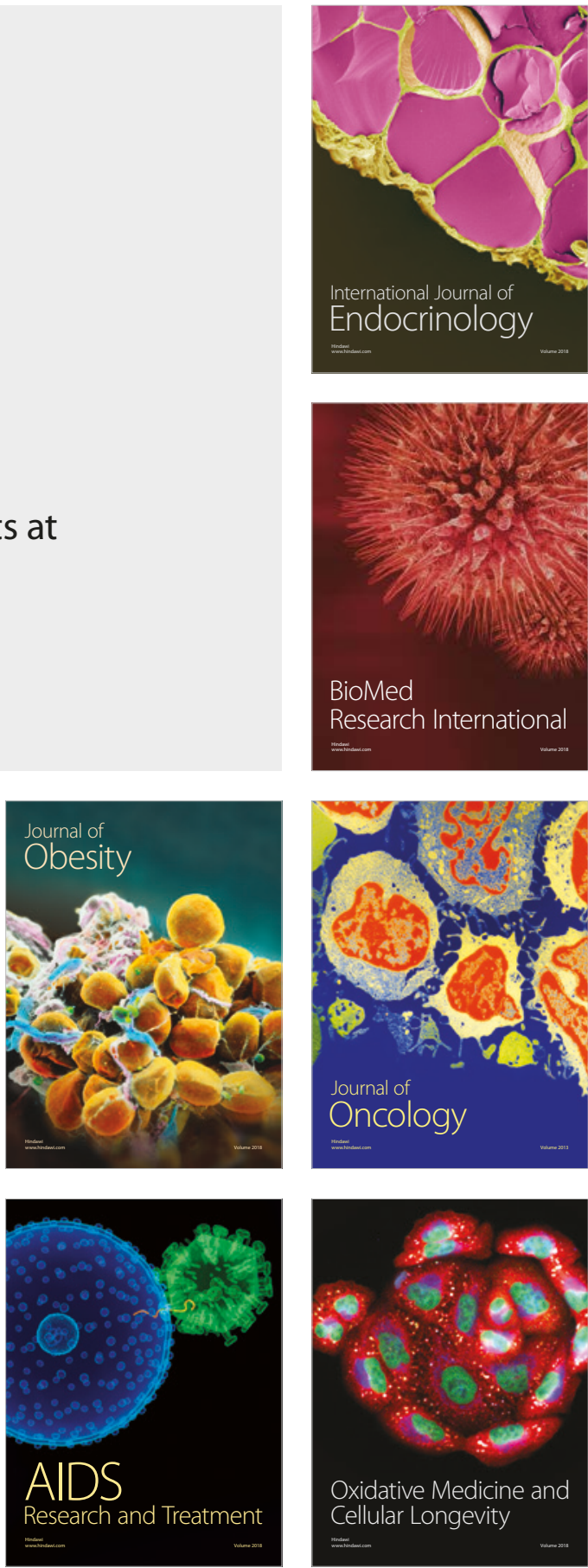